\title{
Polymyxin B-Immobilized Fiber Column Direct Hemoperfusion for Micro-Preemie Infants with Septic Shock. Is Extended Duration Better than Early Start? Comment to the Letter to the Editor of Nishizaki and Colleagues
}

\author{
Chieko Mitaka $^{\text {a }}$ Toshiaki lba $^{b}$ Claudio Ronco ${ }^{c}$ \\ aDepartment of Anesthesiology and Pain Medicine, Juntendo University Faculty of Medicine, Tokyo, Japan; \\ ${ }^{b}$ Department of Emergency Medicine and Disaster Medicine, Juntendo University Graduate School of Medicine, \\ Tokyo, Japan; 'Department of Nephrology Dialysis and Transplantation, International Renal Research Institute, \\ Vicenza, Italy
}

\section{Dear Editor,}

We thank Nishizaki and colleagues [1] for referring to our article [2] and reporting the use of polymyxin Bimmobilized fiber column direct hemoperfusion (PMXDHP) therapy for micro-preemie infants in their letter to the editor. Our article demonstrated that longer duration of PMX-DHP was associated with improved hemodynamics and pulmonary oxygenation in adult patients with septic shock. There is a physical difference between micro-preemie infants and adult patients. We also think that strategy for micro-preemie must be considered separately from adult finding in the treatment of septic shock. Therefore, we would like to make a few comments on PMX-DHP treatment in neonates with septic shock.

The Japan Society for Neonatal Health and Development issued a guideline for PMX-DHP therapy for infants/neonates in Japanese in 2010. PMX-DHP is indicated for pediatric patients with septic shock when the patients manifest endotoxemia associated with the criteria for pediatric sepsis [3]. The criterion for excluding PMX-DHP therapy for pediatric patients with septic shock is intracranial bleeding or tendency to bleed in general. The following points are important for PMX-DHP treatment in neonates: (1) an appropriately sized catheter should be inserted for vascular access; (2) an appropriately sized PMX column should be used; (3) the blood flow rate should be gradually increased to $2-5 \mathrm{~mL} / \mathrm{kg} / \mathrm{min}$ to prevent circulatory collapse; (4) nafamostat mesylate should be infused at a rate of $0.5-1.0 \mathrm{mg} / \mathrm{kg}$ per hour for anticoagulant and thereafter maintained at the rate necessary to sustain activated clotting time for 150-200 s. When the priming volume is $>10 \%$ of the patient's body weight, a mixture of a red blood cell suspension and physiological saline is needed to prevent anemia. The PMX$01 \mathrm{R}$ column is usually used for neonates with septic shock because circulating blood volume is low. The PMX-01R column, a lower priming volume $(8 \mathrm{~mL})$, has been used for smaller children (body weight $<10 \mathrm{~kg}$ ).

Yamashita et al. [4] made an in vitro experiment to elucidate whether the PMX-01R column has ability for adsorbing endotoxin for longer treatment. Their study revealed that the PMX-01R column could remove endotoxin for $24 \mathrm{~h}$. Although it is theoretically possible to extend the duration of PMX-DHP treatment in neonates, it will be practically difficult to perform longer treatment in neonates because of unstable hemodynamics during PMX treatment.
(C) 2021 The Author(s)

Published by S. Karger AG, Basel

This is an Open Access article licensed under the Creative Commons Attribution-NonCommercial-4.0 International License (CC BY-NC) (http://www.karger.com/Services/OpenAccessLicense), applicable to the online version of the article only. Usage and distribution for commercial purposes requires written permission.
Correspondence to:

Chieko Mitaka, c-mitaka@juntendo.ac.jp 
Endotoxin triggers a signaling cascade for leukocytes, macrophages, and endothelial cells to secrete various mediators including cytokines, leading to septic shock. Maeda et al. [5] have shown that PMX-DHP treatment decreased the interleukin- 6 level from $\geq 1,000 \mathrm{pg} / \mathrm{mL}$ to $<500 \mathrm{pg} / \mathrm{mL}$ in 8 premature infants with systemic inflammatory response syndrome. Nishizaki and colleagues [1] reported in the letter to the editor that prognosis of septic neonates in earlier PMX-DHP induction was better than that in later PMX-DHP induction. The reason of this finding may be due to earlier reduction in the cytokine level by earlier PMX-DHP induction, resulting in prevention of cytokine storm. Therefore, earlier PMX-DHP induction might be effective in hemodynamically unstable neonates with septic shock.

Ventricular tachycardia or ventricular fibrillation may occur in consequence of low circulating volumes several minutes after the initiation of extracorporeal circulation in neonates. Therefore, physicians should be careful of their lower circulating blood volumes during PMX-DHP treatment. We believe that PMX-DHP is a useful therapy in neonates with septic shock.

\section{Conflict of Interest Statement}

The authors have no conflicts of interest to declare.

\section{Funding Sources}

The authors did not receive any funding.

\section{Author Contributions}

C.M. drafted the manuscript, and T.I. and C.R. reviewed the manuscript. All authors read and approved the final manuscript.

\section{References}

1 Nishizaki N, Obinata K, Shimizu T. Polymyxin B-Immobilized Fiber Column Direct Hemoperfusion for micro-preemie infants: Is extended duration better than early start? Blood Purif.

2 Mitaka C, Kusaoi M, Kawagoe I, Satoh D, Iba T. Impact of extended duration of polymxin B-immobilized fiber column direct hemoperfusion on hemodynamics, vasoactive substance requirement, and pulmonary oxygenation in patients with sepsis: an observational study. Blood Purif. 2021 Apr;28:1-8.

3 Goldstein B, Giroir B, Randolph A; International Consensus Conference on Pediatric Sepsis. International pediatric sepsis consen- sus conference: definitions for sepsis and organ dysfunction in pediatrics. Pediatr Crit Care Med. 2005;6(1):2-8.

4 Yamashita C, Moriyama K, Hasegawa D, Kato Y, Sakai T, Kawaji T, et al. In vitro study of endotoxin adsorption by a polymyxin B-immobilized fiber column. Blood Purif. 2018; 46(4):269-73.

5 Maeda Y, Ibara S, Tokuhisa T, Ishihara C, Hirakawa E, Matsui T, et al. Polymyxin B-immobilized fiber column direct hemoperfusion and continuous hemodiafiltration in premature neonates with systemic inflammatory response syndrome. Pediatr Int. 2016;58(11) $1176-8$ 